\title{
Fructose-a double edged sword for cell death versus differentiation and long-term survival of NSC-34 motor neuron like cells in vitro
}

\section{Divya Lodha}

Center for Preclinical and Translational Medical Research (CPTMR), Central Research Facility, Sri Ramachandra Institute of Higher Education and Research

Jamuna R. Subramaniam ( $\boldsymbol{\sigma}$ jamuna17@sriramachandra.edu.in )

Center for Preclinical and Translational Medical Research (CPTMR), Central Research Facility, Sri Ramachandra Institute of Higher Education and Research

\section{Research Article}

Keywords: Fructose, NSC-34, differentiation, motor neurons, cell proliferation, neurites, ROS, nrf-2, SOD1, cell death

Posted Date: February 9th, 2021

DOI: https://doi.org/10.21203/rs.3.rs-181783/v1

License: (c) (i) This work is licensed under a Creative Commons Attribution 4.0 International License. Read Full License 


\section{Abstract}

In various neurological and neurodegenerative diseases (ND), motor neurons (MN) of the spinal cord are affected leading to movement impairments. The ND, Amyotrophic Lateral Sclerosis (ALS), is caused due to $\mathrm{MN}$ degeneration. ALS afflicts athletes and other major sports personalities, who generally consume fructose enriched sports drinks. Recently, we have reported that high fructose (F5\%) impairs the metabolic activity in the NSC-34, MN cell line and reduces the healthspan of $C$. elegans. But how fructose impacts the MNs either in vitro or in vivo in the long term is not understood. Here we report, to our surprise, that high fructose ( $\mathrm{F} 5 \%)$ treatment of NSC-34 leads to differentiation of $1-2 \%$ of cells with progressive neurite extension. They could be maintained for 80 days in vitro with $5 \% \mathrm{CO}_{2}$ and $\mathrm{O}_{2}$ at $18.8 \%$. On the contrary, $5 \%$ fructose significantly reduced cell viability by $\sim 85 \%$ and inhibited cell proliferation by Day 10 . Nuclear staining displayed multiple nuclei in the cells indicative of cytokinesis inhibition which led to the lack of cell proliferation. Further, F5\% significantly increased ROS levels (^ $34 \%)$, the potential cause for reduced viability. In addition, no induction of expression of the master oxidative stress response regulator, the transcription factor, $n r f-2$, or the downstream effector, sod1, was evident. Despite the adverse effects, in the absence of any, $\mathrm{F} 5 \%$ is a potential strategy to maintain at least a small percentage of MNs for a long time, 45 days in vitro, which also reinforces the Redox-Cell death versus cell survival conundrum.

\section{Introduction}

In recent decades, lifestyle and food intake has drastically changed. Though life expectancy has increased, associated chronic non-communicable diseases like metabolic syndrome, hypertension, diabetes mellitus and neurodegenerative diseases caused due to lifestyle modifications are on the rise ${ }^{1}$. While fructose occurs naturally in fruits in small quantities, its uncontrolled consumption as the inexpensive and highly addictive ${ }^{2}$ refined sugar/high fructose corn syrup, in large quantities found in confectioneries, baked/processed food and sports drinks have meteorically risen in the past two-three decades. Only recently, it has come to light that fructose plays a key role in afflicting most of the world's population with obesity ${ }^{3}$, non-alcoholic fatty liver disease, ${ }^{4,5}$ and the like.

Off - late, fructose is implicated to be involved in various neurological conditions, neuro-inflammation ${ }^{6}$ and neurodegenerative diseases ${ }^{7}$. It has been known for some time now, that fructose can cross the blood-brain barrier in small quantities, and contribute to energy output ${ }^{8}$. Recent studies also suggest that in the event of a surplus of glucose in the brain, it is converted to fructose through the sorbitol pathway and metabolized ${ }^{9}$. In the Neurodegenerative disease (ND), Amyotrophic Lateral Sclerosis (ALS), characterized by motor neuron (MN) degeneration leading to progressive paralysis and eventually death, which is both sporadic and familial, the preponderance is higher in sports personnel ${ }^{10}$. They generally consume large quantities of sports drinks enriched with glucose and fructose. Also, when ALS is caused by $C 9$ orf 72 repeats, fructose metabolism seems to be impaired in the astrocytes of these patients ${ }^{11}$. As in ALS, mobility or motor control is impaired in many NDs like Parkinson's disease, and deciphering the 
mechanism(s) in vivo is a challenge. NSC-34, a mouse spinal cord motor neuron cell-line derived through fusion with neuroblastoma ${ }^{12-15}$, is an in vitro model widely studied.

NSC-34 cells express the neurotransmitter acetylcholine synthesizing enzyme choline acetyltransferase $(\mathrm{ChAT})^{16}$. Under optimal conditions, like the addition of human umbilical cord-derived stromal cells conditioned medium ${ }^{17}$, retinoic acid, ${ }^{18}$ or vascular endothelial growth factor ${ }^{19}$, these cells differentiate to generate neurites as long as $0.6 \mathrm{~mm}$ in vitro ${ }^{17}$. As primary spinal cord motor neuron cultures are challenging to establish and even harder to maintain in vitro for a long term of more than three weeks ${ }^{20}$, NSC-34 is a good cell line model to understand the general effect of fructose and identify the potential mechanisms that bring about the adverse effects.

Reactive oxygen species (ROS) like superoxide, $\mathrm{O}_{2}$; and hydroxyl, $\mathrm{OH} ;$, radicals are produced as byproducts of ATP synthesis through complete oxidation of glucose and other intermediates of metabolism coupled to oxidative phosphorylation in the mitochondria ${ }^{21}$ and are extremely harmful. But, when produced in small amounts in a controlled manner endogenously, ROS regulates multiple protective functions: cell division/differentiation, inflammation, stress response, autophagy, immune function, and other events through signaling cascades ${ }^{22,23}$. ROS can induce neuronal differentiation ${ }^{24,25}$ and can control their plasticity ${ }^{26}$. But endogenous overproduction of ROS or external insult could be extremely toxic resulting in severe pathophysiology. Hence, intricate antioxidant defense systems are in place. The nuclear factor erythroid -2 related factor (Nrf-2), an established regulator of oxidant defense, normally repressed by Keap1, (Kelch-like erythroid cell-derived protein with CNC homology-associated protein 1) upon activation by ROS, dissociates from Keap-1 and induces the expression of antioxidant response element (ARE) dependent genes which inactivate ROS and maintain redox homeostasis of the cells ${ }^{27}$. One of the downstream effectors of $n r f-2$, the enzyme, $\mathrm{Cu} / \mathrm{Zn}$ Superoxide dismutase I (SOD1), which dismutates $\mathrm{O}_{2}{ }^{--}$and converts it to $\mathrm{H}_{2} \mathrm{O}_{2}$ in the cytoplasm and mitochondrial intermembrane space ${ }^{28}$, is also implicated in ALS ${ }^{16}$. Since fructose plays a major role in energy metabolism and the generation of ROS ${ }^{29,30}$, its effects on the oxidative stress response need to be better understood. Also, fructose effects on neuronal differentiation and long-term maintenance are not known.

Hence, in this report, we address the effects of fructose on NSC-34 cell proliferation, differentiation, neurite extension, maintenance for a long term, and the potential contribution of ROS in cell proliferation inhibition and/or induction of differentiation.

\section{Materials And Methods}

\section{Cell Culture}

Neuroblastoma x mouse spinal cord motor neuron cell line (NSC-34) was maintained using standard protocols as reported earlier 7,16,17 in Dulbecco's modified Eagle medium (DMEM) with 4.5mg/ml glucose supplemented with $10 \%$ Fetal Bovine Serum (FBS) and an antibiotic - antimycotic solution of 
Streptomycin and Penicillin and Amphotericin B. The cells were maintained at $37^{\circ} \mathrm{C}$ with $5 \% \mathrm{CO}_{2}$ and $18.8 \% \mathrm{O}_{2}$ in the Forma series $\mathrm{II} \mathrm{CO}_{2}$ incubator (Thermo Fischer Scientific, Waltham, USA) and sub-cultured every 3-4 days. All the reagents were from Gibco - Life Technologies, Gaithersburg, MD, USA unless otherwise mentioned.

\section{Fructose Treatment}

Fructose at a final concentration of $5 \%(277 \mathrm{mM})$ was added to the cells seeded in T25 flasks $\left(2 \times 10^{5}\right.$ cells) in DMEM complete medium and this was maintained at $37^{\circ} \mathrm{C}$ with $5 \% \mathrm{CO}_{2}$ and $18.8 \% \mathrm{O}_{2}$ for the long-term assay.

Measurement of cell viability.

Thiazolyl Blue Tetrazolium Bromide (MTT) was used to measure cell viability. $5 \times 10^{4}$ cells/well were seeded on 24 well plates and allowed to stabilize overnight. The next day, fructose at a final concentration of $5 \%$ was added to the same high glucose $(4.5 \mathrm{mg} / \mathrm{ml})$ DMEM medium and incubated for various days. After removing the medium at the end of exposure time, the cells were treated with MTT for 3 hours. The medium was replaced with 100\% DMSO (dimethyl sulphoxide), incubated for 30 min at $37^{\circ} \mathrm{C}$, then colorimetric measurement was done at $570 \mathrm{~nm}$. All reagents were from SRL Chemicals, Chennai, India. MTT assay was carried out thrice in duplicates.

\section{Nuclear staining}

Acridine orange $(\mathrm{AO})$ is a nucleic acid dye that permeates the nuclear membrane and emits green fluorescence when bound to dsDNA ${ }^{31}$. For this assay, $2 \times 10^{4}$ cells were seeded onto 24 well plates and treated with fructose $5 \%$ for 48 hours, followed by staining with AO (Sigma Aldrich, Bengaluru, India) at a final concentration of $50 \mu \mathrm{g} / \mathrm{ml}$ for 30 mins at $37^{\circ} \mathrm{C}$. After a couple of $1 \times$ PBS washes, the cells were subject to fluorescence microscopy.

\section{Measurement of cellular ROS levels}

ROS levels were measured by using 2',7'-Dichlorofluorescin diacetate (DCF-DA) (Sigma Aldrich, Bengaluru, India). A previously established protocol was used ${ }^{32}$. Roughly, $5 \times 10^{4}$ cells/well were seeded on 24 well plates and allowed to stabilize overnight. Cells were then treated with fructose (F5\%) and incubated for 48 hours, following which the medium was changed to the subsequent DMEM medium with $\mathrm{F} 5 \%$ containing 2',7'-Dichlorofluorescin diacetate (DCF-DA) $(10 \mu \mathrm{m})$ and incubated for one hour at $37^{\circ} \mathrm{C}$. The fluorescence intensity was measured at excitation and emission wavelengths of $485 \mathrm{~nm}$ and $535 \mathrm{~nm}$, respectively. DCF-DA background was subtracted, and the graph was plotted. The assay was done thrice in quadruplicates.

\section{Expression analysis of Oxidative stress response}


Total RNA was extracted from the NSC34 motor neurons using RNA isoplus (TaKaRa Bio, Nojihigashi, Kusatsu, Japan) as reported with minor modifications ${ }^{17}$. This was reverse transcribed using MMLV reverse transcriptase (Thermo Fischer Scientific, Waltham, USA) in the presence of an RNAse inhibitor (Thermo Fischer Scientific, Waltham, USA). The specific genes Nrf-2 (F- $5^{\prime}$

TGTCGCCAGCTCAAGGGCACAGTGC 3'; R- 5' AGGCTCCATCCTCCCGAACC 3', size - 617bps), sod-1 (F- 5' AACCAGTTGTGGTGTCAGGA 3'; R- 5' CTCCTGAGAGTGAGATCACA 3', size - 245bps) and $\beta$-actin (F- 5' AGCCATGTACGTAGCCATCC 3'; R- 5' TCGGAACCGCTCATTGCCG 3', size - 378bps) were amplified by PCR carried out in the ThermoCycler (TaKaRa Bio, Nojihigashi, Kusatsu, Japan). The amplified PCR products were then separated by agarose gel electrophoresis (1-1.5\%).

\section{Microscopy.}

The cells were analyzed for nuclear staining in Nikon Eclipse-Ti fluorescence microscope, using FITC filter. The nucleus was viewed with $10 x$ and $40 x$ objectives. The same microscope was utilized for viewing and capturing the phase-contrast images. The images were obtained with a CCD camera and Qimaging software.

\section{$\underline{\text { Statistics }}$}

Mean and standard deviation were calculated, and significance was estimated by t-tests using GraphPad Prism ver. 8.2.1 (GraphPad Software, La Jolla, California, USA).

\section{Results}

Induction of motor neuron differentiation and long-term survival

High fructose $(5 \%)$ treatment, in the presence of $18.8 \% \mathrm{O}_{2}$ provided through oxygen inflow in addition to the standard cell culture conditions of $37^{\circ} \mathrm{C}$ and $5 \% \mathrm{CO}_{2}$, induced differentiation in $1-2 \%$ of the cells in the motor neuron cell line NSC-34 (Fig. 1A). Moreover, extensive neurite extension and branching was evident in the differentiated cells, with the neurites growing into long and thick processes as time progressed (Fig.1) (3-4weeks DIV). Although fructose is reported to cause cell death ${ }^{7,33}$, high fructose exposure supported the survival of $\sim 0.5 \%$ of cells as differentiated neurons till $\sim 80$ days (Fig. 2A-F). This survival was evident only in the presence of $5 \%$ fructose with $18.8 \% \mathrm{O}_{2}$ supplementation.

$\underline{\text { Inhibition of cell proliferation }}$

Treatment of the NSC-34 cell line with high fructose (5\%) abrogated cellular proliferation as early as day 5 (Fig. 3A). This was more obvious on days 10 and 15 (Fig. 3A). MTT assays revealed a significant reduction in viable cells, by $53 \%$ due to fructose exposure on day 5 (Fig. 3B) and $\sim 85 \%$ by Day 10 [Day $5^{*_{-}}$ $\mathrm{P}=0.017$; Day $10 * \star \star-\mathrm{P}=0.0007$ ] compared to control cells.

Disruption of cell proliferation through suppression of cell division 
Although high fructose treated cells showed visible division of the nucleus (Fig. 4) cytoplasmic division was absent which resulted in multinucleation. This was evident even in some of the differentiated motor neuron-like cells (Fig. 4A). Staining with Acridine orange on day 20 revealed two or more well-divided nuclei (Fig.4B). While normal cell division was imminent in the control cells (Fig. 5B.a) it was lacking in F5\% treated cells. The cells seemed to be arrested before cytokinesis resulting in multinucleation (Fig. 5B.b-d), and thereby inhibition of cell proliferation.

\section{High fructose induces ROS generation}

Fructose is known to increase ROS levels in various cell types ${ }^{29,30,34}$. To identify fructose mediated ROS generation and its impact on NSC-34 cells, first, ROS levels were measured using the fluorescent dye indicator, DCF-DA. F5\% significantly increased DCF-DA fluorescence intensity by $34 \%$ upon $48 \mathrm{hrs}$ exposure (Fig. 5A), showing a significant increase in ROS levels [ $\left.{ }^{\star \star \star}-\mathrm{P}=0.0002\right]$. The neuronal cell body exhibited extensive vacuolation (Fig. 5B) indicative of ROS mediated cellular stress and toxicity.

\section{Absence of oxidative stress response}

Generally, ROS level increase is accompanied by induction of oxidative stress response. As F5\% increased ROS levels, an increase in the expression of the oxidative stress response genes was investigated. Nrf-2, the major, master inducer of expression of a multitude of oxidative stress response pathway genes 27 including sod-1, an enzyme responsible for conversion of superoxide into less toxic hydrogen peroxide, and oxygen ${ }^{28}$ was determined through RT-PCR. Though both nrf-2 and sod 1 were expressed upon F5\% treatment, the expression level was the same as in control (Fig. 5C). This lack of induction of expression will result in an abysmal oxidative stress response to protect against increased ROS due to high fructose treatment.

\section{Discussion}

In the past few decades, diet and lifestyle have changed drastically. One of the major changes is the addition of fructose, like refined sugar or high fructose corn syrup, being used widely and indiscriminately in processed/baked confectioneries. Fructose usage is more than that of glucose or sucrose, due to its ready availability. Reports of fructose's role in major diseases like diabetes mellitus ${ }^{35}$, obesity 3,36 , nonalcoholic fatty liver disease ${ }^{4,5,30}$, cancer ${ }^{37}$, and even in neurological/ neurodegenerative diseases including $\mathrm{ALS}^{38}$ is unsettling. ALS is more prevalent among sports personnel. Energy beverages consumed by them as an immediate source of muscular energy are supplemented with fructose. But fructose's effect on motor neuron degeneration which brings about ALS in sportspeople is unclear. Hence, we set out to address fructose impact on the NSC-34 MN line in vitro. Further, oxygen uptake is quite different in physically active people (sports), with high $\mathrm{VO}_{2 \max }$ (maximal oxygen uptake) ${ }^{39}$. To closely mimic this, unlike normal mammalian cell culture, the oxygen level was maintained at $18.8 \%$, with exclusive $\mathrm{O}_{2}$ supply in addition to $5 \% \mathrm{CO}_{2}$. In vivo $\mathrm{O}_{2}$ level is below $6.5 \%$ in most of the tissues including brain and muscle ${ }^{40}$. But, that of the spinal cord is not known. 
Earlier, we reported that similar to high glucose being toxic ${ }^{41}$, exposure of NSC-34 cells to $5 \%$ fructose for 48 hrs caused $\sim 60 \%$ cell death ${ }^{42}$ even without oxygen (18.8\%) supplementation. Presently, F5\% showed $\sim 85 \%$ cell death on day 10 (Fig. 3). Unexpectedly, around $1-2 \%$ of the cells differentiated with long, branched neurites and could be maintained in vitro up to 80 days (Fig.1\&2) only with $\mathrm{O}_{2}$ supplementation. The caveat here is that routine $\mathrm{CO}_{2}$ incubator oxygen level is supposed to be around $18.5 \%$ (present in the air) even without $\mathrm{O}_{2}$ supplementation ${ }^{410}$. High fructose treatment combined with oxygen supplementation provides a strategy for long-term maintenance of differentiated spinal cord motor neurons which is hard to achieve. A further detailed study will provide novel insights into the motor neuron function and abnormalities.

Earlier we reported that high fructose exposure for a short term (6-48hrs) disrupted mitochondrial function and metabolic activity ${ }^{42}$ in NSC-34 cells and shortened the healthspan of $C$. elegans. Here, we noticed NSC-34 cells with multiple nuclei in the presence of F5\% (Fig. 4), while well-divided cells are evident (Fig.4B) in the control. Somehow, high fructose inhibits only cytokinesis, thereby hindering cell proliferation leading to multinucleated cells. One possibility is the inefficient synthesis of the cellular components needed due to reduced or lack of ATP production ${ }^{43}$ upon F5\% treatment.

Generally, endogenously produced ROS is regulated to activate signaling cascades to induce a multitude of protective functions. But at higher doses, ROS induces cellular toxicity. Fructose is known for increasing ROS production in many cellular models: hepatocytes ${ }^{30}$, adipocytes ${ }^{43}$, myocytes ${ }^{44}$, and cardiomyocytes ${ }^{45}$. Similarly, here high fructose increased ROS levels by $35 \%$ (Fig. 5A). Strangely the oxidative stress response machinery through $n r f-2$ was not induced (Fig. $5 C$ ). Besides, multiple vacuoles were observed (Fig.6B) in some of the cells as seen with superoxide/hydrogen peroxide exposure ${ }^{46}$ or in the overall/motor neurons vacuolation of the spinal cord in transgenic ALS model mice expressing mutant (G93A/G37R) human Cu/Zn SOD1 ${ }^{47,48}$.

Overall, high fructose is detrimental, and caution is required against its unfettered intake as part of the diet. At the same time, it provides a strategy or direction, in combination with oxygen supplementation, to differentiate and maintain motor neurons for a long term in vitro which can open new avenues on multiple fronts given the severity and lack of treatment for several neurological, neurodegenerative and neuromuscular diseases.

\section{Declarations}

\section{Acknowledgment}

Divya Lodha thanks Sri Ramachandra Institute of Higher Education and Research for the Chancellor's Fellowship. The authors thank the Center for Stem Cell and Regenerative Medicine and the Center for Indian System of Medicine, Sri Ramachandra Institute of Higher Education and Research, for the infrastructure. 
No ethical clearance is required.

The authors declare no conflict of interest.

\section{References}

1. Bidwell, A. Chronic Fructose Ingestion as a Major Health Concern: Is a Sedentary Lifestyle Making It Worse? A Review. Nutrients 9, 549 (2017).

2. Lustig, R. H. Fructose: it's 'alcohol without the buzz'. Adv. Nutr. Bethesda Md 4, 226-235 (2013).

3. Tappy, L. \& Lê, K.-A. Metabolic Effects of Fructose and the Worldwide Increase in Obesity. Physiol. Rev. 90, 23-46 (2010).

4. Ter Horst \& K.W., Serlie, M.J. Fructose Consumption, Lipogenesis, and Non-Alcoholic Fatty Liver Disease. Nutrients 9, 981 (2017).

5. Moore, J., Gunn, P. \& Fielding, B. The Role of Dietary Sugars and De novo Lipogenesis in NonAlcoholic Fatty Liver Disease. Nutrients 6, 5679-5703 (2014).

6. Harrell, C. S. et al. High-fructose diet during adolescent development increases neuroinflammation and depressive-like behavior without exacerbating outcomes after stroke. Brain. Behav. Immun. 73, 340-351 (2018).

7. Mastrocola, R., Nigro, D., Cento, A. S., Chiazza, F., Collino, M.\& Aragno, M. High-fructose intake as risk factor for neurodegeneration: Key role for carboxy methyllysine accumulation in mice hippocampal neurons. Neurobiol. Dis. 89, 65-75 (2016).

8. Thurston, J. H., Levy, C. A., Warren, S. K. \& Jones, E. M. Permeability of the blood-brain barrier to fructose and the anaerobic use of fructose in the brains of young mice. J. Neurochem. 19, 16851696 (1972).

9. Xu, J. et al. Elevation of brain glucose and polyol-pathway intermediates with accompanying braincopper deficiency in patients with Alzheimer's disease: metabolic basis for dementia. Sci. Rep. 6, 27524 (2016).

10. Blecher, R. et al. Contact Sports as a Risk Factor for Amyotrophic Lateral Sclerosis: A Systematic Review. Glob. Spine J. 9, 104-118 (2019).

11. Allen, S. P. et al. C9orf72 expansion within astrocytes reduces metabolic flexibility in amyotrophic lateral sclerosis. Brain J. Neurol. 142, 3771-3790 (2019).

12. Cashman, N. R. et al. Neuroblastoma $\times$ spinal cord (NSC) hybrid cell lines resemble developing motor neurons. Dev. Dyn. 194, 209-221 (1992).

13. Durham, H. D., Dahrouge, S. \& Cashman, N. R. Evaluation of the spinal cord neuron X neuroblastoma hybrid cell line NSC-34 as a model for neurotoxicity testing. Neurotoxicology 14, 387-395 (1993). 
14. Richardson, K. et al. The Effect of SOD1 Mutation on Cellular Bioenergetic Profile and Viability in Response to Oxidative Stress and Influence of Mutation-Type. PLOS ONE 8, e68256 (2013).

15. Chen, T., Turner, B. J., Beart, P. M., Sheehan-Hennessy, L., Elekwachi, C., \& Muyderman, H. Glutathione monoethyl ester prevents TDP-43 pathology in motor neuronal NSC-34 cells. Neurochem. Int. 112, 278-287 (2018).

16. Thangavelu, S. R., Tripathi, P. P., Arya, U., Mishra, H. K. \& Subramaniam, J. R. ALS associated mutant SOD1 impairs the motor neurons and astrocytes and wild type astrocyte secreted-factors reverse the impaired motor neurons. Ann. Neurosci. 18, 48-55 (2011).

17. Kumar, A., Mishra, H. K., Dwivedi, P. \& Subramaniam, J. R. Secreted trophic factors of Human umbilical cord stromal cells induce differentiation and neurite extension through PI3K and independent of cAMP pathway. Ann. Neurosci. 22, (2015).

18. Piras, S. et al. Monomeric Aß1-42 and RAGE: key players in neuronal differentiation. Neurobiol. Aging 35, 1301-1308 (2014).

19. Kulshreshtha, D. et al. Vascular Endothelial Growth Factor Attenuates Neurodegenerative Changes in the NSC-34 Motor Neuron Cell Line Induced by Cerebrospinal Fluid of Sporadic Amyotrophic Lateral Sclerosis Patients. Neurodegener. Dis. 8, 322-330 (2011).

20. Hanson, M. G., Shen, S., Wiemelt, A. P., McMorris, F. A. \& Barres, B. A. Cyclic AMP elevation is sufficient to promote the survival of spinal motor neurons in vitro. J. Neurosci. Off. J. Soc. Neurosci. 18, 7361-7371 (1998).

21. Yang, S. \& Lian, G. ROS and diseases: role in metabolism and energy supply. Mol. Cell. Biochem. 467, $1-12(2020)$.

22. Ristow, M. \& Schmeisser, S. Extending life span by increasing oxidative stress. Free Radic. Biol. Med. 51, 327-336 (2011).

23. Brieger, K., Schiavone, S., Miller, F. J. \& Krause, K.-H. Reactive oxygen species: from health to disease. Swiss Med. Wkly. 142, w13659 (2012).

24. Suzukawa, K. et al. Nerve growth factor-induced neuronal differentiation requires generation of Rac1regulated reactive oxygen species. J. Biol. Chem. 275, 13175-13178 (2000).

25. Munnamalai, V. \& Suter, D. M. Reactive oxygen species regulate F-actin dynamics in neuronal growth cones and neurite outgrowth. J. Neurochem. 108, 644-661 (2009).

26. Tsatmali, M., Walcott, E. C., Makarenkova, H. \& Crossin, K. L. Reactive oxygen species modulate the differentiation of neurons in clonal cortical cultures. Mol. Cell. Neurosci. 33, 345-357 (2006).

27. Ma, Q. Role of nrf2 in oxidative stress and toxicity. Annu. Rev. Pharmacol. Toxicol. 53, 401-426 (2013).

28. Sun, Y. et al. ROS systems are a new integrated network for sensing homeostasis and alarming stresses in organelle metabolic processes. Redox Biol. 37, (2020).

29. Cho, Y. E. et al. Fructose Promotes Leaky Gut, Endotoxemia and Liver Fibrosis through CYP2E1Mediated Oxidative and Nitrative Stress. Hepatol. Baltim. Md (2019) doi:10.1002/hep.30652. 
30. Jensen, T. et al. Fructose and sugar: A major mediator of non-alcoholic fatty liver disease. J. Hepatol. 68, 1063-1075 (2018).

31. Darzynkiewicz, Z. Differential staining of DNA and RNA in intact cells and isolated cell nuclei with acridine orange. Methods Cell Biol. 33, 285-298 (1990).

32. Liu, W. et al. Anti-glycation and anti-oxidative effects of a phenolic-enriched maple syrup extract and its protective effects on normal human colon cells. Food Funct. 8, 757-766 (2017).

33. Jaiswal, N. et al. Fructose induces mitochondrial dysfunction and triggers apoptosis in skeletal muscle cells by provoking oxidative stress. Apoptosis 20, 930-947 (2015).

34. Lanaspa, M. A. et al. Uric acid induces hepatic steatosis by generation of mitochondrial oxidative stress: potential role in fructose-dependent and -independent fatty liver. J. Biol. Chem. 287, 4073240744 (2012).

35. Gerrits, P. M. \& Tsalikian, E. Diabetes and fructose metabolism. Am. J. Clin. Nutr. 58, 796S-799S (1993).

36. Tappy, L. Fructose-containing caloric sweeteners as a cause of obesity and metabolic disorders. J. Exp. Biol. 221, (2018).

37. Nakagawa, T. et al. Fructose contributes to the Warburg effect for cancer growth. Cancer Metab. 8, 16 (2020).

38. Poulton, K. R. \& Rossi, M. L. Peripheral nerve protein glycation and muscle fructolysis: evidence of abnormal carbohydrate metabolism in ALS. Funct. Neurol. 8, 33-42 (1993).

39. Burnley, M. \& Jones, A. M. Oxygen uptake kinetics as a determinant of sports performance. Eur. J. Sport Sci. 7, 63-79 (2007).

40. Ast, T. \& Mootha, V. K. Oxygen and mammalian cell culture: are we repeating the experiment of Dr. Ox? Nat. Metab. 1, 858-860 (2019).

41. Chen, F., Qian, L. H., Deng, B., Liu, Z. M., Zhao, Y. \& Le, Y. Y. Resveratrol protects vascular endothelial cells from high glucose-induced apoptosis through inhibition of NADPH oxidase activation-driven oxidative stress. CNS Neurosci. Ther. 19, 675-681 (2013).

42. Lodha, D., Rajasekaran, S., Jayavelu, T. \& Subramaniam, J. R. Detrimental effects of fructose on mitochondria in mouse motor neurons and on C. elegans healthspan. Nutr. Neurosci. (2020) doi:10.1080/1028415X.2020.1853413.

43. Legeza, B., Balázs, Z. \& Odermatt, A. Fructose promotes the differentiation of 3T3-L1 adipocytes and accelerates lipid metabolism. FEBS Lett. 588, 490-496 (2014).

44. Jaiswal, N., Maurya, C. K., Pandey, J., Rai, A. K. \& Tamrakar, A. K. Fructose-induced ROS generation impairs glucose utilization in L6 skeletal muscle cells. Free Radic. Res. 49, 1055-1068 (2015).

45. Takata, T., Sakasai-Sakai, A., Ueda, T. \& Takeuchi, M. Intracellular toxic advanced glycation endproducts in cardiomyocytes may cause cardiovascular disease. Sci. Rep. 9, 2121 (2019).

46. Kazzaz, J. A., Horowitz, S., Li, Y. \& Mantell, L. L. Hyperoxia in Cell Culture: A Non-apoptotic Programmed Cell Death. Ann. N. Y. Acad. Sci. 887, 164-170 (1999). 
47. Wong, P. C. et al. An adverse property of a familial ALS-linked SOD1 mutation causes motor neuron disease characterized by vacuolar degeneration of mitochondria. Neuron 14, 1105-1116 (1995).

48. Subramaniam, J. R. et al. Mutant SOD1 causes motor neuron disease independent of copper chaperone-mediated copper loading. Nat. Neurosci. 5, 301-307 (2002).

\section{Figures}

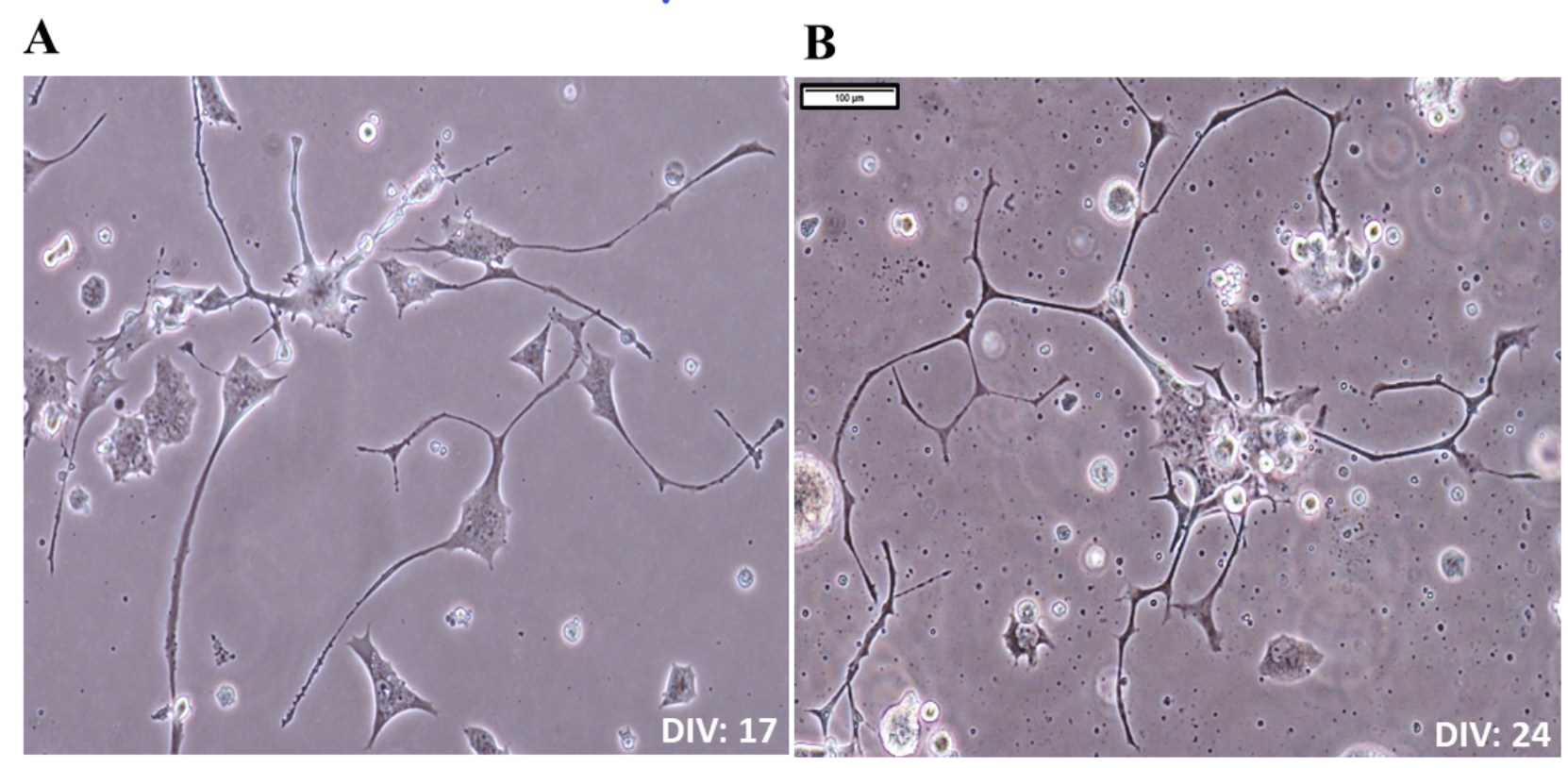

\section{Figure 1}

High fructose induces neuronal differentiation and neurite extension A. Fructose $5 \%$ treatment, induced neuronal differentiation and facilitated neurite formation. B. A single differentiated neuron with long and thick neurites. [Scale: $1 \mathrm{~cm}=100 \mu \mathrm{m}]$ 


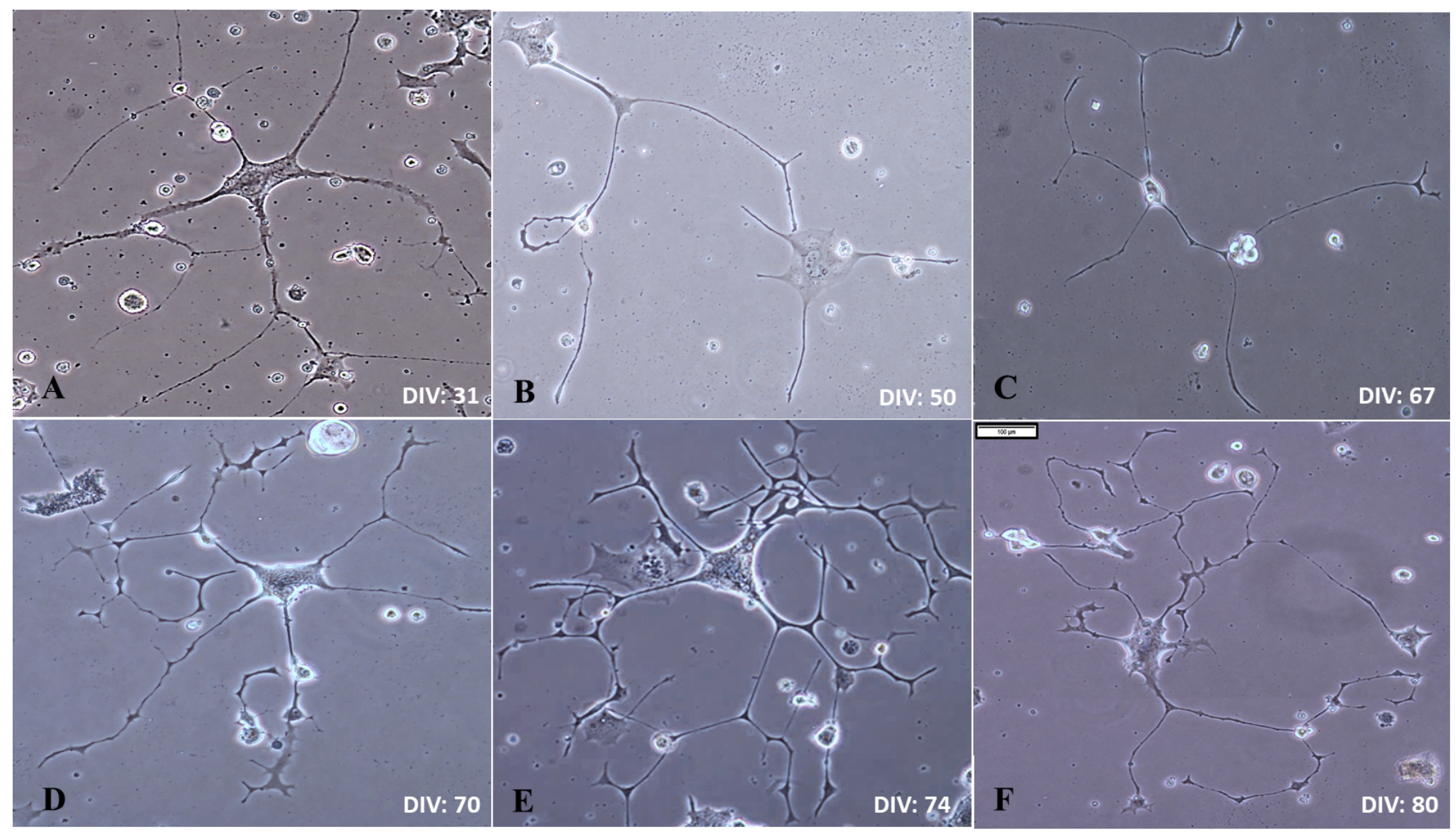

Figure 2

Cells exposed to high fructose survive for extensively long periods A - F. Differentiated cells with extended neurites. DIV- No. of days in in vitro in culture). [Scale: $1 \mathrm{~cm}=100 \mu \mathrm{m}$ ]

A

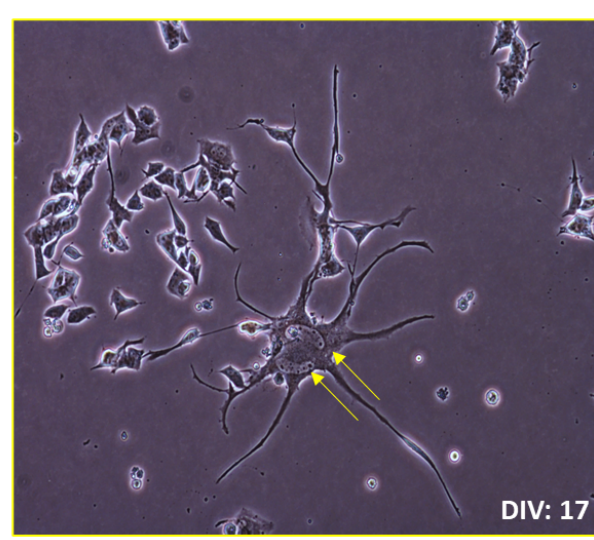

B

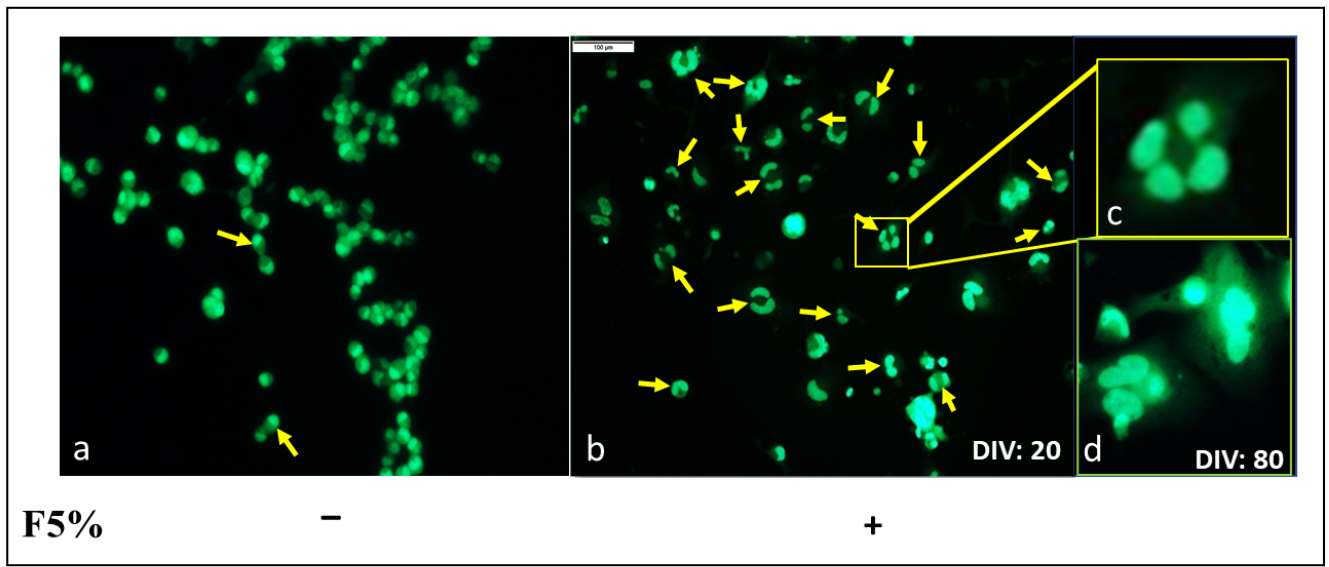

\section{Figure 3}

High fructose inhibits cell proliferation A. Deterred cell density with $5 \%$ fructose treatment as early as day 5. [Scale: $1 \mathrm{~cm}=100 \mu \mathrm{m}$ ] B. Significant cell death confirmed by MTT assay performed at day 5 and day 10. [Day $5 *_{-} P=0.0275 ;$ Day $10 * * *_{-} P<0.0001$ ] 
A

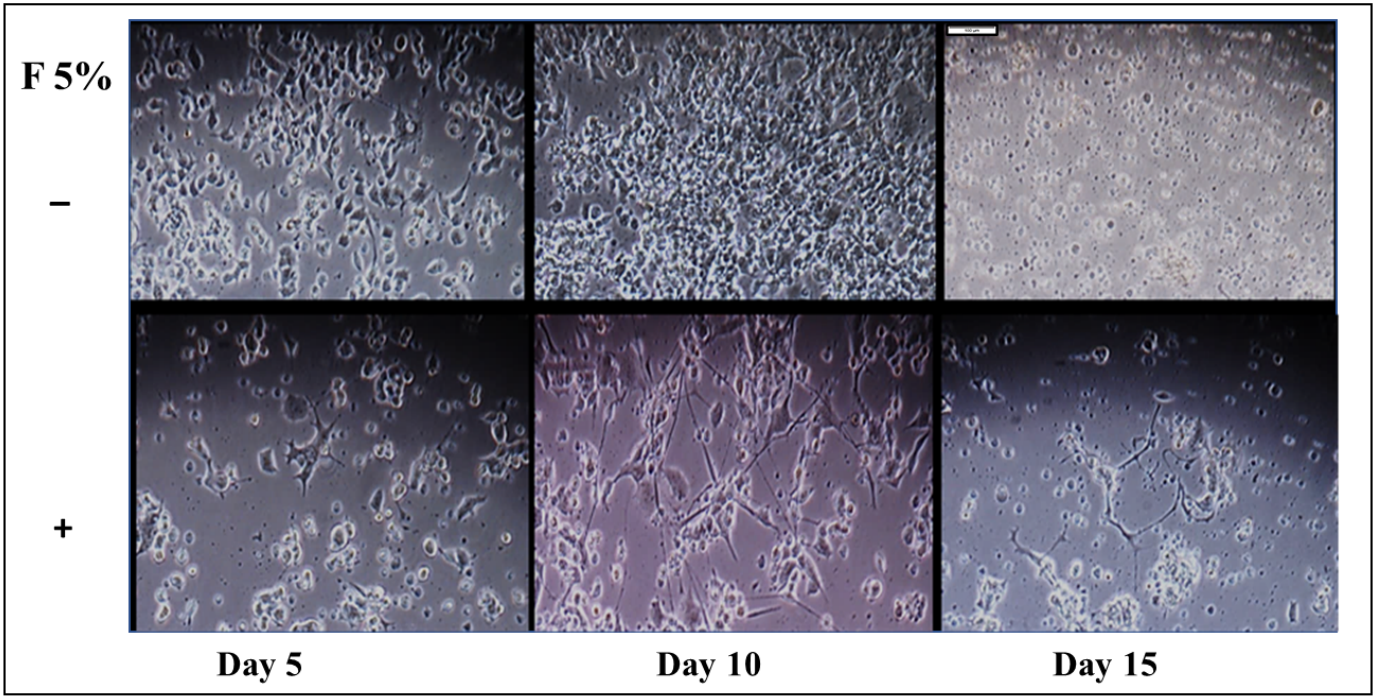

B

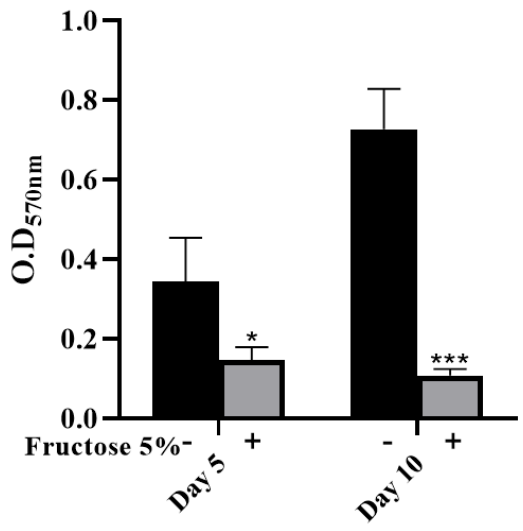

Figure 4

Multinucleation observed in most cells exposed to high fructose A. Fructose interfered with cell division. Binucleated differentiated cell. B. 5\% Fructose inhibited cell division at cytokinesis. a. Upon acridine orange staining, control cells show normal cytokinesis each divided cell with a single nucleus. b. $5 \%$ fructose led to bi-nucleated, tri-nucleated, and poly-nucleated cells. c. Four nuclei (magnified inset). [Scale: $1 \mathrm{~cm}=100 \mu \mathrm{m}]$. Nuclei are indicated with yellow arrows.
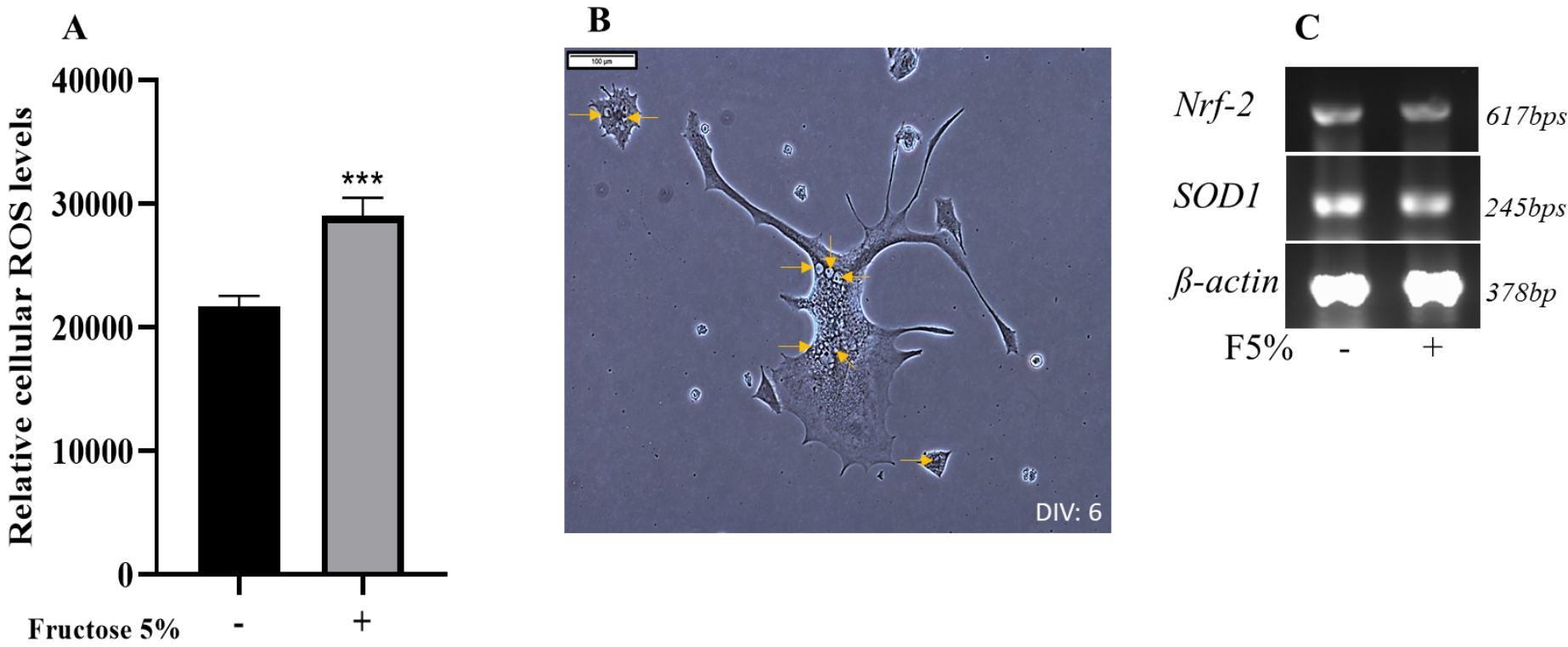

\section{Figure 5}

Neuronal ROS levels increase after high fructose treatment A. DCF-DA - fluorescence intensity measured ROS increased significantly ( 50\%) in neurons exposed to high fructose for 48 hours. [ $* \star *_{-} P=0.0002$ ] B. Vacuoles (black arrows) in the cells exposed to high fructose. [Scale: $1 \mathrm{~cm}=100 \mu \mathrm{m}] \mathrm{C}$. Expression of nrf- 
2 and sod 1 in NSC-34 cells by RT-PCR. (-) - control; (+) - F5\% treated; The levels are not altered with F5\% exposure. 